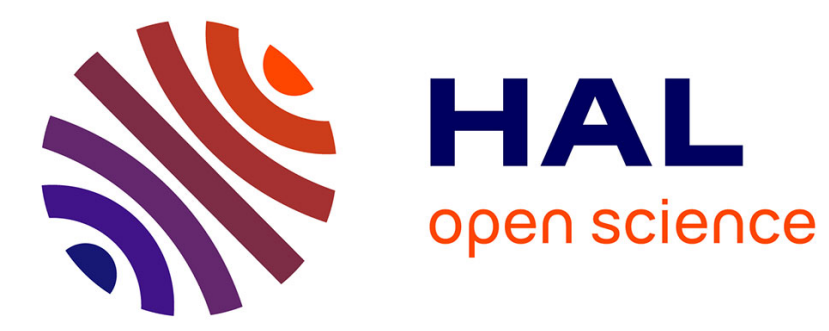

\title{
Towards an Accurate Tracking of Liver Tumors for Augmented Reality in Robotic Assisted Surgery
}

Nazim Haouchine, Jérémie Dequidt, Igor Peterlik, Erwan Kerrien, Marie-Odile Berger, Stéphane Cotin

\section{- To cite this version:}

Nazim Haouchine, Jérémie Dequidt, Igor Peterlik, Erwan Kerrien, Marie-Odile Berger, et al.. Towards an Accurate Tracking of Liver Tumors for Augmented Reality in Robotic Assisted Surgery. International Conference on Robotics and Automation (ICRA), Jun 2014, Hong Kong, China. hal-01003262

\section{HAL Id: hal-01003262 \\ https://hal.inria.fr/hal-01003262}

Submitted on 10 Jun 2014

HAL is a multi-disciplinary open access archive for the deposit and dissemination of scientific research documents, whether they are published or not. The documents may come from teaching and research institutions in France or abroad, or from public or private research centers.
L'archive ouverte pluridisciplinaire HAL, est destinée au dépôt et à la diffusion de documents scientifiques de niveau recherche, publiés ou non, émanant des établissements d'enseignement et de recherche français ou étrangers, des laboratoires publics ou privés. 


\title{
Towards an Accurate Tracking of Liver Tumors for Augmented Reality in Robotic Assisted Surgery
}

\author{
Nazim Haouchine ${ }^{1}$, Jeremie Dequidt ${ }^{1}$, Igor Peterlik ${ }^{2}$, Erwan Kerrien ${ }^{3}$ \\ Marie-Odile Berger ${ }^{3}$ and Stephane Cotin ${ }^{1}$
}

\begin{abstract}
This article introduces a method for tracking the internal structures of the liver during robot-assisted procedures. Vascular network, tumors and cut planes, computed from preoperative data, can be overlaid onto the laparoscopic view for image-guidance, even in the case of large motion or deformation of the organ. Compared to current methods, our method is able to precisely propagate surface motion to the internal structures. This is made possible by relying on a fast yet accurate biomechanical model of the liver combined with a robust visual tracking approach designed to properly constrain the model. Augmentation results are demonstrated on in-vivo sequences of a human liver during robotic surgery, while quantitative validation is performed on an ex-vivo porcine liver experimentation. Validation results show that our approach gives an accurate surface registration with an error of less than $6 \mathrm{~mm}$ on the position of the tumor.
\end{abstract}

\section{INTRODUCTION}

Considerable recent advances in medicine have made Minimally Invasive Surgery (MIS) a well-established procedure for hepatic surgery, with strong benefits for patients. However, the reduced endoscopic field of view, the lack of depth and tactile perception make hepatic resection and tumor removal a major challenge that requires advanced surgical skills. Augmented Reality (AR) techniques have the potential to counteract these regressions and provide an enriched visual feedback by the seamless integration of intraoperative images and any targets or risk structures, such as tumors or vessels, visible in 3D pre-operative imaging (cf Figure 1).

Developing a full AR system for MIS implies solving a number of difficult problems, ranging from instrument tracking to intuitive augmented visualization [1]. Among the most difficult and still unsolved issues is the capacity to tackle, in real time, large elastic deformations on the targeted organ. Though the problem of elastic registration or tracking has been largely addressed in the medical community, elastic deformation in AR systems is only addressed, to our knowledge, in a very limited number of papers, and mainly applied to 3 organs: kidney, heart and liver.

Kidneys do not actually undergo elastic deformations during surgery but rather move independently from their

\footnotetext{
${ }^{1}$ Nazim Haouchine, Jeremie Dequidt and Stephane Cotin are with INRIA and Lille University, France nazim.haouchinedinria.fr, jeremie.dequidt@inria.fr, stephane.cotineinria.f

${ }^{2}$ Igor Peterlik is with Image-guided Minimally Invasive Surgery Institute, IHU Strasbourg, France peterlik@gmail.com

${ }^{3}$ Erwan Kerrien and Marie-Odile Berger are with INRIA and Lorraine University, Nancy, France erwan.kerrien@inria.fr, marie-odile.berger@inria.fr
}

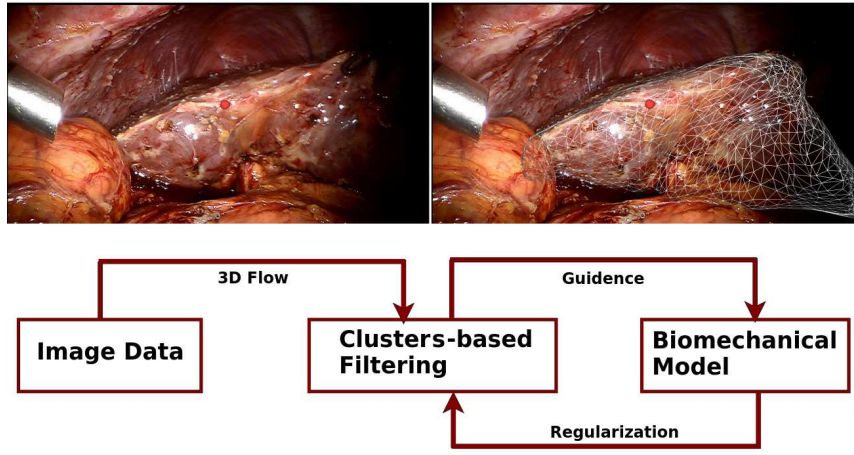

Fig. 1. Augmented Liver Tumor during Minimally Invasive Surgery. Top: The registration of the liver mesh including the tumor onto endoscopic images. Bottom: Computational flow of the method.

surrounding tissues. As such, related works resort to a rigid 3D-3D registration e.g. based on implanted markers [2] or an Iterative Closest Point (ICP) surface matching [3]. Recently Puerto-Souza et al. [4] proposed a Hierarchical MultiAffine matching algorithm capable of long-term tracking for augmentation during partial nephrectomy. This approach estimates a set of affine transformations from clusters of features in order to relocate occluded or missing features to obtain a coherent spatio-temporal mesh registration. Figl et al. [5] also express the non-rigid registration problem as a rigid registration between stereoscopic views and a 4D model of the heart built from 4D scan data, thus leveraging the cyclicity of the organ motion. A 4D scan of the heart is also used in [6], but is coupled with a biomechanical model to relate the surface motion to internal forces. A local tuning of the deformation is therefore possible and the surface deformation is correctly propagated to in-depth invisible structures. No periodic or rigid pattern can be relied upon in the liver case. Unpredictable, possibly large, deformations can be observed. Furthermore, volume tracking is required since we are interested in in-depth augmentation whereas many approaches are dedicated to the tracking of surfaces. In a similar context, Kim et al. [7] propose a solution that is both applicable in a monoscopic setup and integrates a robust image feature reconstruction approach. But their method only retrieves surface deformation and cannot ensure an accurate augmentation of inner structures.

Mechanical-based deformable models have proven to be relevant for volume deformation since they allow to define elastic properties of the shape and thus to infer in-depth 
structure motion [8], [9], [10]. Registration is done either by solving mechanical equations given image constraints provided by reconstructed 3D points [8] or using the concept of active models where an energy is minimized which takes into account the internal behavior of the model -through the biomechanical constraints- and external image constraints that measure the adequation between the model and the recovered 3D data [9], [10].

The work presented here is an improvement of the former work on AR for deformable organs [13], [12]. In these works, a method employing a biomechanical model guided by a stereo-based tracking algorithm which is able to interpolate in the volume surface deformations was proposed. The physical model used, provably adapted to the liver [11], is superimposed in real-time onto the laparoscopic images within tumors. In this paper we mainly focus on the reconstruction and the selection of $3 \mathrm{D}$ points which are used to guide the physical model. In particular, techniques based on moving least-squares are used to improve the set of recovered 3D points. We also restrict the set of points which are used to guide the mechanical model using clustering techniques with significant impact on the accuracy and the robustness of the method. With respect to existing works, we improve upon [8] by proposing a method that does not demand a computationally expensive stereo surface recovery and upon [4] by performing a robust and long-term tracking which is able to handle elastic deformations of the organ. The validation of our approach is presented on both in vivo and ex vivo data where we report visually accurate registration on images of an actual laparoscopic procedure and show that the error between the estimated and actual positions of an artificial tumor implanted in a porcine liver is less than $6 \mathrm{~mm}$.

\section{STEREOSCOPIC FLOW ESTIMATION}

The use of a biomechanical model to propagate the surface motion to the internal structures not visible on the endoscopic images, such as tumors, requires a stable tracking sufficiently robust with respect to the image noise. For that purpose, we propose a new tracking method designed to guide the physically-based model. Our tracking method follows these steps:

- Sparse three-dimensional recovery from stereoscopic images.

- Moving Least-Squares-based outliers filtering and surface approximation.

- Control point selection with clustering and temporal flow estimation.

\section{A. Sparse three-dimensional recovery}

Intra-abdominal surface reconstruction has been under investigation in several research groups (for a complete survey, please refer to [17]). The methods range from passive techniques [19], [21] based only on endoscopic image informations, to active techniques [20], [22] that involve hardware modifications. Our method relies on a passive approach using a stereo endoscope, calibrated according to Zhang's [18] approach. Features are detected in each image

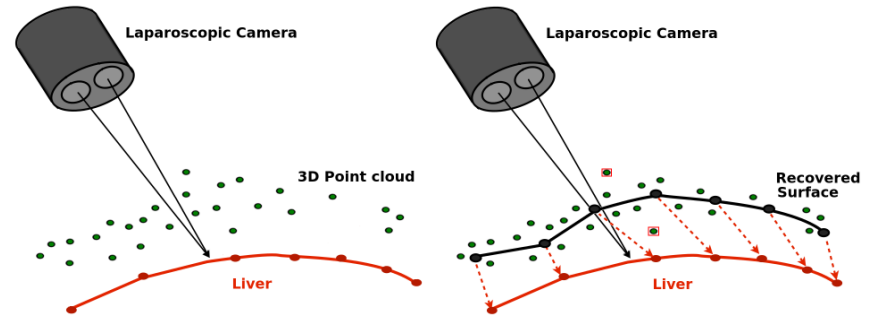

Fig. 2. MLS-based surface recovery. Left: Three-dimensional sparse point cloud recovery. Right: outliers filtering and surface reconstruction using MLS, the control points selected after clustering are registered to the biomechanical model of the liver

pair (rectified for distortion) using the Speeded-Up Robust Features (SURF) descriptor and are tracked over time thanks to the Lucas-Kanade optical flow method, this coupling has proven to be optimal for laparoscopic surgery [14]. Feature correspondences between stereo images are obtained with a nearest neighbour criterion on feature descriptors coupled with the epipolar constraint. A sparse set of $m$ 3D points, denoted by $3 \times m$ coordinate vector $\mathbf{z}$, is reconstructed by triangulation.

Examples of 2D matched features and reconstructed 3D points from laparoscopic images are shown in Figure 3.

\section{B. Moving Least-Squares-based surface reconstruction}

The sparse set $\mathbf{z}$ of recovered $3 \mathrm{D}$ points may contain erroneous points. In order to remove these points and to obtain a smoother point set $\mathbf{z}^{\prime}$ we take benefits of the MLS surface to approximate a surface and to detect the noisy points.

Moving Least-Squares surface (MLS) is a mesh-free, nonparametric method to approximate a surface from a set of unstructured scattered point introduced by [16].

To determine the MLS surface given a point set $P=\left\{p_{i} \in \mathbb{R}^{3}\right\}$, a projection procedure which projects any points near the point set onto the surface using a local approximation is conducted. Given a point $r \in \mathbb{R}^{3}$, the process follows two steps. First the computation of a local patch, then the projection of the query point $r$ onto the surface using a polynomial function :

- Compute a local reference plane in the neighborhood of $r$ : Find a plane $H$ with normal vector $a \in \mathbb{R}^{3}$ passing through some point $q=r+t a$ (for some $t \in \mathbb{R}$ ) such that $\|a\|=1$ and $H$ minimizes the least-squares error:

$$
\sum_{p_{i} \in P}<a, p_{i}-q>^{2} \theta\left(\left\|p_{i}-q\right\|\right)
$$

Here, $\left\langle a, p_{i}-q\right\rangle^{2}$ is the squared distance from each point $\left\{p_{i}\right\}$ to the plane $H$, and $\theta\left(\left\|p_{i}-q\right\|\right)$ is the weighting function, usually a Gaussian.

- Project $r$ on the surface : We consider the points $x_{i}$ to be the orthogonal projections of the points $p_{i}$ on the plane $H$ with projected distance $f_{i}=\left\langle a, p_{i}-q\right\rangle$ oriented with normal $a$ and origin $q$. Thus, we apply the MLS 

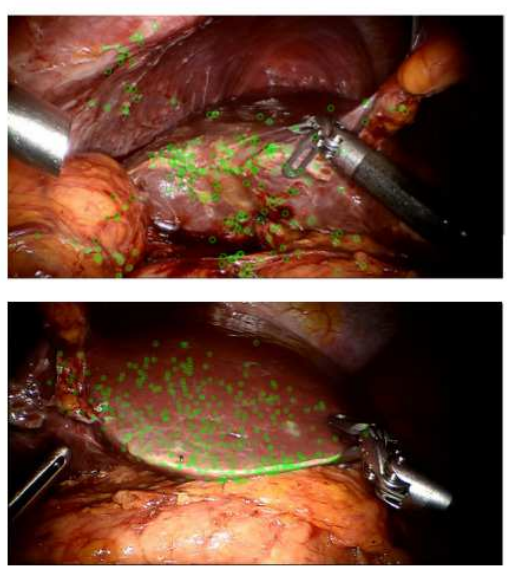

(a)

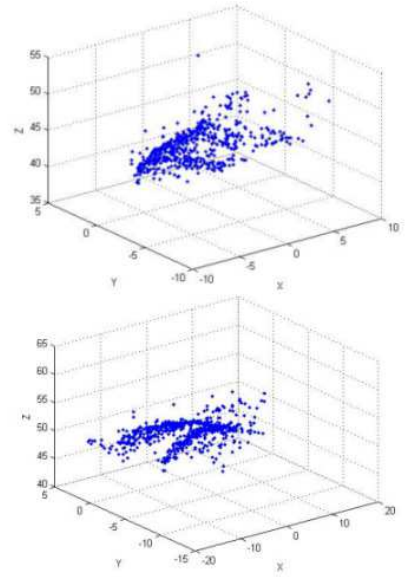

(b)

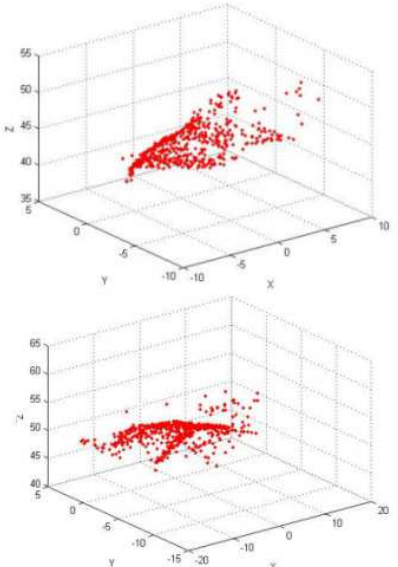

(c)

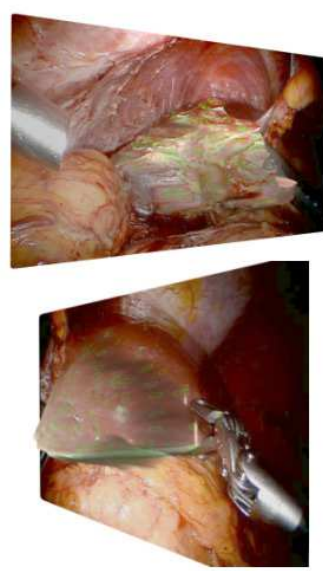

(d)

Fig. 3. Stereoscopic flow estimation on laparoscopic images of the abdominal cavity showing a part of the liver in two different cases with (top) high textured liver and (bottom) low textured liver: (a) Original input images, (b) the resulting sparse 3D point set $\mathbf{z}$ from stereo matching, (c) the resulting sparse 3D point set $\mathbf{z}^{\prime}$ from MLS outliers filtering, (d) the projected image on the reconstructed surface.

function approximation which consists of construction of a bivariate polynomial $\tilde{g} \in \mathbb{R}^{3}$ of degree $m$ (here we take $m=3$ ) which locally minimizes the least-squares error around $q$ :

$$
\sum_{p_{i}}\left(g\left(x_{i}\right)-f_{i}\right)^{2} \theta\left(\left\|p_{i}-q\right\|\right)
$$

That amounts to project $r$ along the normal with distance $\tilde{g}(0)$ (local approximation of the surface at $q$ ).

\section{Clustering-based Filtering}

The clustering-based filter $(\mathbf{C b F})$ permits to create a set of $n$ 3D control points, denoted by $3 \times n$ coordinate vector $\mathbf{y}$ that guides the biomechanical model. Instead of constraining the model directly using the forces generated from feature displacements, the clustering calculates a displacement field more suitable to handle large deformations (see section IV for more details).

First a set of control point is built by downsampling a set $\mathbf{z}^{\prime}$ of 3D points resulting from MLS step. Each control point defines a region of interest to group the surrounded features. The nearest features to the control point are assigned to the cluster by combining the Hessian responses of the SURF detector and the Shepard's Inverse Distance Weighting (IDW) following the formula $D(p)=q_{p} \sum_{i=1}^{k} W_{i} d_{i}$ where $D$ is the weighted displacement of the control point $p, k$ the total number of neighbors and $d_{i}$ the displacement of the feature $i$. Assuming the quality is isotropic at each feature, we define a normalized measure of quality $q_{p}$ for each control point as the average of the SURF Hessian responses of its neighbors. $W_{i}$ is the weight assigned to each feature and is as follows:

$$
W_{i}=\frac{\left(\frac{R_{c}-h_{i}}{R_{c} h_{i}}\right)^{2}}{\sum_{j=1}^{k}\left(\frac{R_{c}-h_{j}}{R_{c} h_{j}}\right)^{2}}
$$

$R_{c}$ is the radius of the cluster in 3D space, $h_{i}$ the distance of the feature from the control point and $k$ the total number of neighbors.
The Shepard's IDW allows the nearby feature to the control point to have the largest weight while the Hessian response allows to prevent from poor measurement during the tracking.

\section{BIOMECHANICAL MODEL AND NON-RIGID REGISTRATION}

\section{A. Biomechanical model}

We employ a biomechanical model of liver composed of parenchyma and vascular network which is an important source of heterogeneity and anisotropy. From a mechanical standpoint, the parenchyma model relies on a linear tetrahedral finite element modeling using a co-rotational approach [15]. This model is very well suited to handle large displacements and has been proven to be relevant in the context of real-time laparoscopic surgery [11]. The vascular system is modeled with serially linked beam elements previously used in modeling catheters and guide wires model proposed by Duriez et al. [24]. Both models share very similar properties which permit to build the composite model. A more detailed description of both models and the coupling can be found in [13]. Elastic forces are then defined as in [15]:

$$
f_{i}(x)=R_{e} K\left(R_{e}^{\top} x-x^{0}\right)
$$

where $K$ represents the stiffness matrix, $R_{e}$ the corotational matrix and $\mathbf{x}, \mathbf{x}^{\mathbf{0}}$ are vectors of size $3 n$ representing the position of the $n$ degrees of freedom of the mechanical model, respectively at any time $t$ and time $t=0$. It is worth mentioning that our framework was built using flexible and modular software design principles allowing to easily replace the physics-based model.

\section{B. Initial registration}

The initial registration is an important step for AR systems. In this work this step is done manually through a 3D interface as in similar systems [4], [8], [5], [6], [12]. First, the biomechanical model is aligned on the first pair 

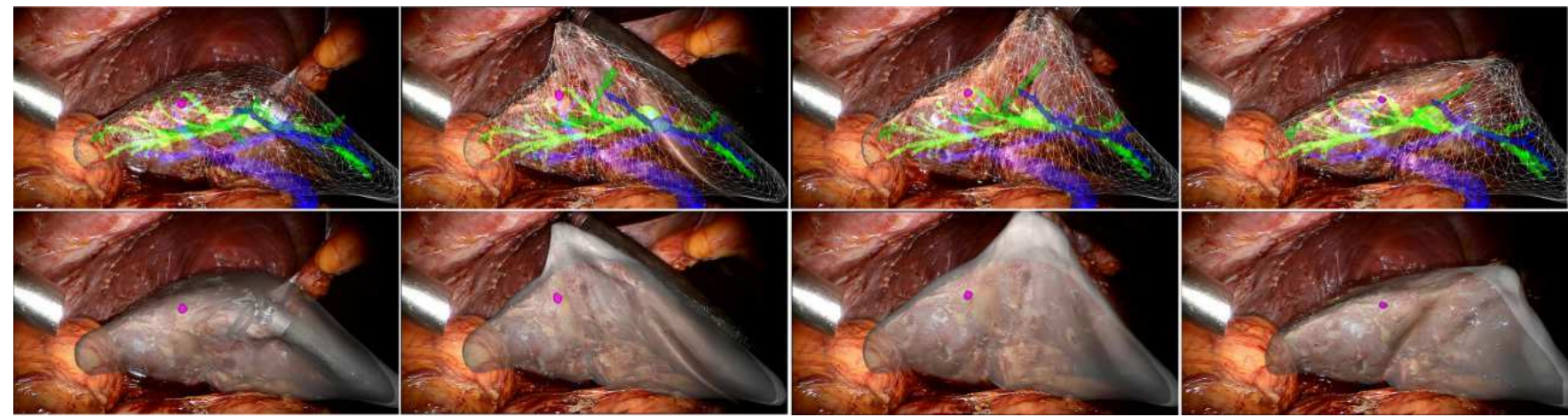

Fig. 4. Selected frames of a superimposition of the biomechanical model onto the human liver during Minimally Invasive Surgery. Top: Heterogeneous model including the parenchyma (white), the portal vein (red), the hepatic vein (blue) and a tumor (sphere). Bottom: A different visualisation of the superimposition

of laparoscopic stereo images. Then, only the reconstructed points that reproject onto the model in the images are kept to filter in points belonging to the liver.

\section{Temporal non-rigid registration}

We propose to handle the non-rigid registration by adding external stretching forces introduced via tracking of 3D control points. The tracked 3D control point set $\mathbf{y}$ represents how visible parts of the liver are moving.

External forces are defined by pairing the $m$ 3D control points $\mathbf{y}$ to the $n$ degrees of freedom $\mathbf{x}$ of the bio-mechanical model. At initialization $(t=0)$, the tracked points $\mathbf{y}^{\mathbf{0}}$ are expressed in barycentric coordinates of the adjacent degrees of freedom leading to the linear relation: $y^{0}=J x^{0}$, where $J$ is a rectangular $(3 m, 3 n)$ matrix. We assume this linear relation remains valid during the deformation.

At any later time $t$, the stretching forces induced by the tracking are defined as $f_{t}(y)=k\left(y-y^{0}\right)$ where $k$ can be seen as the stiffness.

Finally, the stretching forces can be expressed with respect of the degrees of freedom as

$$
f_{t}(x)=J^{\top} f_{t}(y)=f_{t}(x)=J^{\top} k \cdot J\left(x-x^{0}\right)
$$

the parameter $k$ is set to be close to the Young's modulus in order to provide an equilibrium between the stretching forces and the elastic forces (a higher value of $k$ favors stretching forces).

\section{EXPERIMENTATIONS AND RESULTS}

In this section, we present the experimentations conducted to validate our approach. The validation in context of MIS is usually a challenging task. In our case, we propose two validation protocols. The first one is performed in the context of in-vivo human liver surgery, the other involves experiments on ex-vivo porcine liver. In latter case, the results are evaluated within a known ground truth. We also compare our method CbF (tracking with Clusters-based Filtering) with the related work DT (a direct tracking used in [13]).

\section{A. Results on in vivo Human Liver}

The purpose of conducting an in vivo experimentation is to employ our technique in a real-life situation during an abdominal hepatic surgery. Several important disturbing factors are present: the illumination produced by the laparoscopic light, motion of instruments during the intervention and motion of the organ induced by respiration and cardiac cycle.
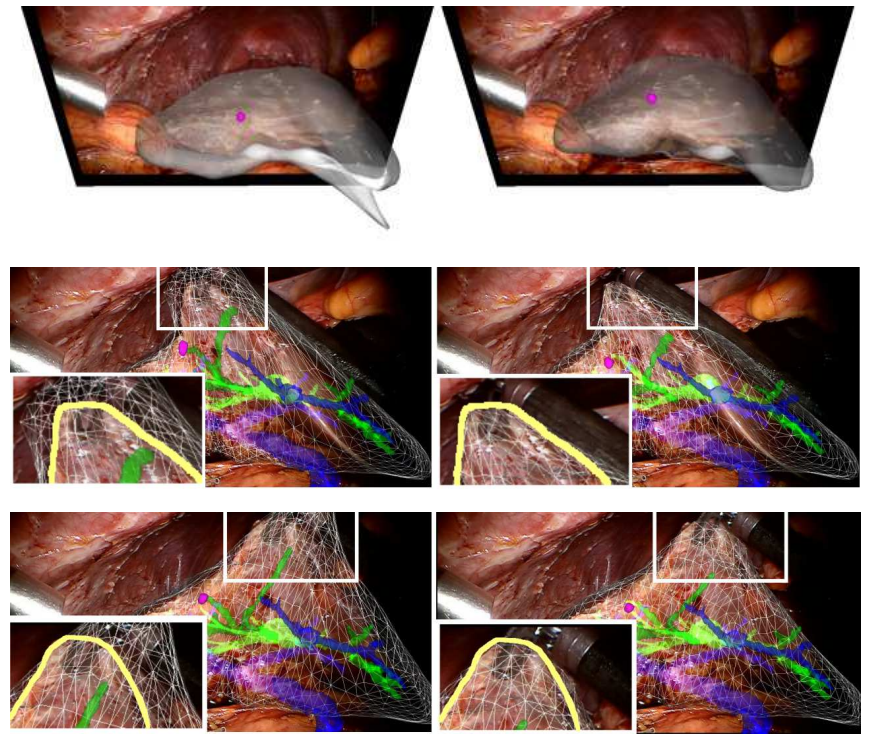

Fig. 5. Comparison between (right) our $\mathbf{C b F}$ tracking and (left) a the DT method. Top: A top view showing the registration error that may occur using a direct method. The biomechanical model (in white wireframe) is locally constrained by a 3D feature in the case of a direct tracking while it is constrained by set of features grouped around a control point. Middle and Bottom: A front view showing the visual accuracy of our method. The correct shape is well recovered even if the boundary (manually segmented and represented here in yellow) is not correct everywhere.

For this study we used a heterogeneous biomechanical model, that takes into account the hepatic and portal vein to describe the liver soft-tissue behaviour, with the following elastic parameters (according to [11]): Young's modulus of parenchyma $E_{p}=7 \mathrm{kPa}$ and Poisson's ratio $v=0.45$, Young's modulus of vessel wall: $E_{v}=0.62 \mathrm{MPa}$ 
and Poisson's ratio $v=0.45$, thickness of the vessel wall: $t_{V}=200 \mu \mathrm{m}$. The parenchyma model was composed of 3391 linear tetrahedral elements and the vascular model of 120 beam elements. The augmentation is achieved in real-time at around $25 \mathrm{fps}$. It's worth mentioning that the real-time achievement is directly related to the number of elements (a larger number of elements will increase the computation time).

Following [14], the parameters of the optical flow consist of $51 \times 51$ window and an interframe motion threshold of 20 pixels. The camera used is the Da Vinci Robot Endoscopic Camera (Intuitive Surgical) with a baseline of about $6 \mathrm{~mm}$, acquiring image of $960 \times 540$ at $29.97 \mathrm{fps}$. On the set of 264 extracted features 26 control points are built when using a radius $R_{c}=0.5 \mathrm{~mm}$.

\begin{tabular}{lcccc} 
& \multicolumn{4}{c}{ Projection error $(\boldsymbol{p x})$} \\
\hline Frame & $\mathbf{\# 6 0}$ & $\mathbf{\# 1 2 0}$ & $\mathbf{\# 1 8 0}$ & $\mathbf{\# 2 4 0}$ \\
\hline Clusters-based Filter & 54.72 & 110.57 & 145.33 & 75.55 \\
\hline Tracking of [13] & 809.95 & 659.04 & 304.78 & 766.01 \\
\hline
\end{tabular}

TABLE I

A COMPARISON BETWEEN THE CLUSTERS-BASED APPROACH AND THE DIRECT TRACKING.

The Figure 4 illustrates four selected frames of the augmentation performed on a sequence of $\mathbf{8 1 2}$ frames showing the long-term robustness of the tracking.

We can notice in the comparison illustrated in Figure 5 that the DT approach may fail to accurately register the model onto the image, mainly because of noisy 3D features that locally constraints the model. The table I summarizes the projection errors of the mesh on the endoscopic images (manually extracted contour) and shows that the CbF tracking permits to reduce considerably the error w.r.t the DT approach.

\section{B. Ex vivo quantitative validation on a porcine liver}

The purpose of our tracking method is to estimate the location of tumors that are not visible in the endoscopic images. To validate such an approach we propose to perform CT scans of the liver before and after a deformation while acquiring the motion with a stereoscopic camera illustrated in Figure 7. Using a wire attached to one lobe of a porcine liver, we move the tissue in order to induce a deformation which is captured with an endoscopic camera ensuring a CTscanning without producing artefact.

We introduce two artificial tumors in two different locations (cf Figure 6), near from the tension zone (tumor (a) with an average size of $8 \mathrm{~mm}$ ) and far from the tension zone (tumor (b) with an average size of $5 \mathrm{~mm}$ ).

In Figure 8 our $\mathbf{C b F}$ tracking is compared with the DT approach using the data acquired during the manipulation of ex-vivo liver. We measure the following quantities: The tumor displacement due to the deformation for the Tumor (a) is $88.6 \mathrm{~mm}$ and Tumor (b) is $26.9 \mathrm{~mm}$. The measured distance between the centers of mass of the ground truth

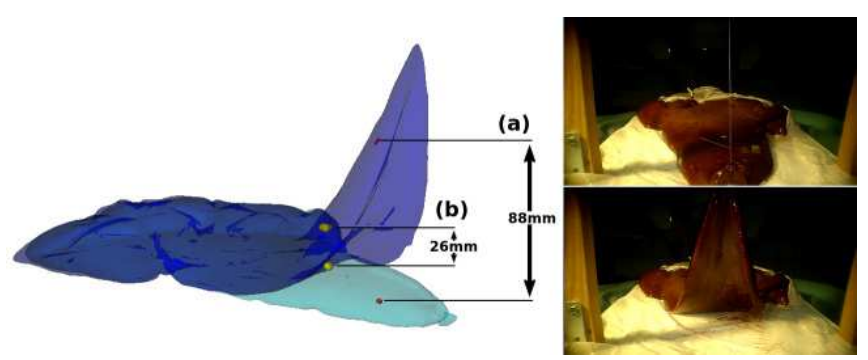

Fig. 6. Computed meshes form scanned porcine liver with the tumors (left) and the corresponding endoscopic images (right).

and the tracked tumors is $\mathbf{5 . 1 3} \mathbf{~ m m}$ for the tumor (a) and $2.20 \mathbf{m m}$ for the tumor (b) when using the $\mathbf{C b F}$ tracking and $39.04 \mathrm{~mm}$ for the tumor (a) and $4.55 \mathrm{~mm}$ for the tumor (b) when using the DT approach.

It represents a significant improvement over the DT method and over current laparoscopic procedures where a safety margin of $10 \mathrm{~mm}$ to $25 \mathrm{~mm}$ is usually considered around the location of the tumor (planned on pre-operative data) [23].

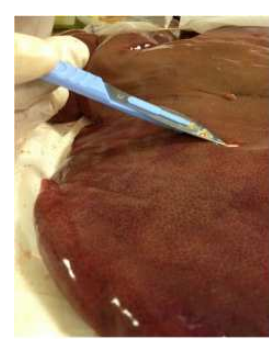

(a)

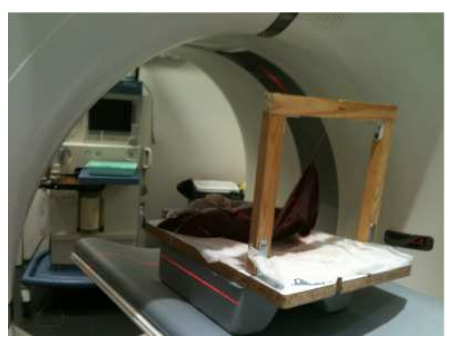

(c)

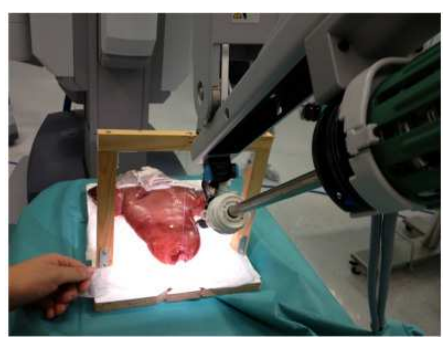

(b)

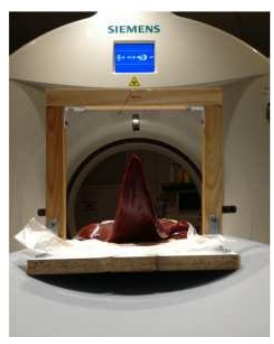

(d)
Fig. 7. Experimental setup for the quantitative validation: (a) insertion of an artificial tumor into one lobe of the liver, (b) a view of the the Da Vinci Robot Camera capturing liver motion, (c) the wire hooked to the lobe is pulled and maintained to perform a CT scan, (d) front view of the CT-scan and the liver.

\section{CONCLUSiON}

One of the current deadlocks to break before AR systems can find their place in the clinical routine of MIS procedures is the ability to capture organs deformations during surgery. Large and unpredictable liver deformation that occur during surgery highly impacts the location of internal structures such as tumors and vessels. We have presented in this paper, an accurate tracking algorithm designed to constrain properly a biomechanical model that is able to capture such deformations. The model deformation is guided by both internal 


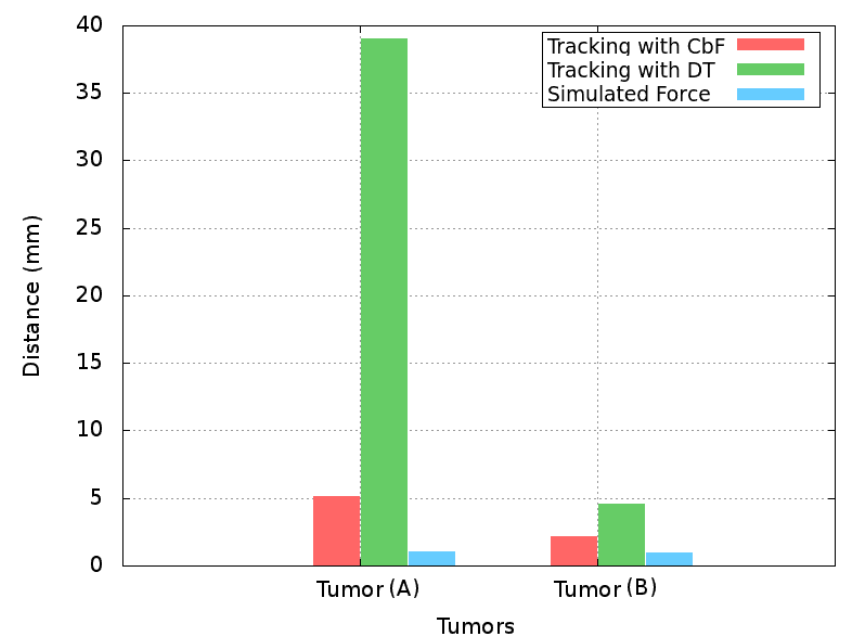

Fig. 8. A comparison study between the $\mathbf{C b F}$, the DT approach and a computer-generated force, on the tumor (a) and tumor (b). The clustering reduces considerably the error between the predicted tumor and the ground truth.

forces, generated by a biomechanically grounded model of the liver, and external forces, that emanate from 3D control points. These 3D control points are built form stereo images through a clusters-based filtering that restrict the noisy image measurement. Such characteristics make our approach able to accurately register the organ surface and inner the deformation motion to the internal structures. Promising results were obtained through in-vivo experimentation on a human liver and ex-vivo validation on a porcine liver where errors well below the current surgical margins and current methods were measured. Future work will address the issue of initialisation that is currently done manually, and the robustness of our approach against actual surgical events such as smoke, bleeding, or sudden instrument occlusions that may hinder the tracking. More validation is obviously required though it is worth mentioning that validation implying actual organs are seldom reported in previous works.

\section{ACKNOWLEDGEMENT}

The authors highly acknowledge the help provided by Mario Sanz Lopez during the experimentations and advisory provided by Dr. Patrick Pessaux.

\section{REFERENCES}

[1] Nicolau S., Soler L., Mutter D., Marescaux J.: Augmented reality in laparoscopic surgical oncology. Journal of Surgical Oncology; 20 (3): $189-201$

[2] Teber D., Guven S., Simpfendorfer T., Baumhauer M., Guven E. O., Yencilek F., Gozen A. S., Rassweiler J.: Augmented reality : a new tool to improve surgical accuracy during laparoscopic partial nephrectomy Preliminary in vitro and in vivo results. Journal of European Urology (2009).

[3] Su L.M. , Vagvolgyi B., Agarwal R., Reiley C. E., Taylor R., Hager G. D : Augmented Reality During Robot-assisted Laparoscopic Partial Nephrectomy: Toward Real-Time 3D-CT to Stereoscopic Video Registration. Journal of Urology, 73(4):896-900, 2009.

[4] Puerto-Souza, G.A. and Mariottini, G.L.: An augmented-reality system for laparoscopic surgery robust to complete occlusions and fast camera motions. In International Conference on Robotics and Automotion 2013.
[5] Figl M., Rueckert D., Hawkes D., Casula R., Hu M., Pedro O., Zhang D. P., Penney G., Bello F., Edwards P.: Image guidance for robotic minimally invasive coronary artery bypass. In Proceedings of the 4th international workshop on Medical Imaging and Augmented Reality (Berlin, Heidelberg, 2008), MIAR '08, Springer-Verlag, pp. 202209.

[6] Pratt P., Visentini Scarzanella M., Stoyanov D. and Yang G-Z.: Dynamic Guidance for Robotic Surgery using Image-Constrained Biomechanical Models. In Medical Image Computing and Computer Assisted Interventions (MICCAI10), 2010.

[7] Jae-Hak Kim , Adrien Bartoli, Toby Collins and Richard Hartley. Tracking by Detection for Interactive Image Augmentation in Laparoscopy. In Workshop on Biomedical Image Registration, WBIR'2012.

[8] Speidel S., Roehl S., Suwelack S., Dillmann R.: Intraoperative surface reconstructionand biomechanical modeling for soft tissue registration. In SCATh joint Workshop on New Technologies for Computer/Robot Assisted Surgery, 2011.

[9] Shen T., Li H.; Huang X.: Active Volume Models for Medical Image Segmentation. In IEEE Trans. Med. Imaging. 30 (3), 2011.

[10] Sermesant M., Forest C., Pennec X., Delingette H, Ayache N.: Deformable biomechanical models: Application to 4D cardiac image analysis. In Medical Image Analysis, 4 (7), 2003.

[11] Peterlik I., Duriez C., Cotin S.: Modeling and Real-Time Simulation of a Vascularized Liver Tissue. In MICCAI 2012.

[12] Haouchine N., Dequidt J., Berger M.-O., and Cotin S. Deformationbased Augmented Reality for Hepatic Surgery. In Medicine Meets Virtual Reality, MMVR 20, San Diego, USA, 2013.

[13] Haouchine N., Dequidt J., Peterlik I., Kerien E., Berger M.-O., and Cotin S. Image-guided Simulation of Heterogeneous Tissue Deformation for Augmented Reality during Hepatic Surgery. In International Sympsium of Mixed and Augmented Reality (to appear), ISMAR'13, Adelaide, Australia, 2013.

[14] Elhawary H., Popovic A.: Robust feature tracking on the beating heart for a robotic-guided endoscope. Int J Med Robot. 2011 Dec;7(4):45968. doi: 10.1002/rcs.418. Epub 2011 Oct 7.

[15] Müller M., Dorsey J., McMillan L., Jagnow R. and Cutler B.: Stable real-time deformations. In ACM SIGGRAPH/Eurographics symposium on Computer Animation 2002.

[16] Levin, D. Mesh-independent surface interpolation. In "Geometric Modeling for Scientific Visualization" Edited by Brunnett, Hamann and Mueller, Springer-Verlag, 2003, 37-49.

[17] L. Maier-Hein, P. Mountney, A. Bartoli, H. Elhawary, D. Elson, A. Groch, A. Kolb, M. Rodrigues, J. Sorger, S. Speidel, D. Stoyanov, Optical techniques for 3D surface reconstruction in computer-assisted laparoscopic surgery, Medical Image Analysis, Volume 17, Issue 8, December 2013, Pages 974-996, ISSN 1361-8415.

[18] Z. Zhang. A flexible new technique for camera calibration. IEEE Trans. Pattern Anal. Mach. Intell., 22(11):13301334, Nov. 2000.

[19] D. Stoyanov. Stereoscopic scene flow for robotic assisted minimally invasive surgery. In Proceedings of the 15th international conference on Medical Image Computing and Computer-Assisted Intervention, MICCAI12, pages 479486. Springer-Verlag, 2012.

[20] X. Maurice, P. Graebling, and C. Doignon. Real-time structured light coding for adaptive patterns. Journal of Real-Time Image Processing, pages 110, 2011.

[21] R. Richa, A. P. L. B, and P. Poignet. Robust 3d visual tracking for robotic-assisted cardiac interventions. In Proceedings of the 13th international conference on Medical image computing and computerassisted intervention, pages 267274. Springer-Verlag, 2010.

[22] Herrera, S.E.M.; Malti, A.; Morel, O.; Bartoli, A., "Shape-fromPolarization in laparoscopy," Biomedical Imaging (ISBI), 2013 IEEE 10th International Symposium on , vol., no., pp.1412,1415, 7-11 April 2013 doi: 10.1109/ISBI.2013.6556798

[23] T. Mala, B. Edwin, I. Gladhaug, E. Fosse, O. Sreide, A. Bergan, and O. Mathisen. A comparative study of the short-term outcome following open and laparoscopic liver resection of colorectal metastases. Surgical Endoscopy And Other Interventional Techniques, 16(7):10591063, 2002.

[24] C. Duriez, S. Cotin, J. Lenoir, and P. Neumann. New approaches to catheter navigation for interventional radiology simulation. Computer Aided Surgery, 2006. 